\title{
miRNA-1271 inhibits cell proliferation in neuroglioma by targeting fibronectin 1
}

\author{
JIAN GONG $^{1}$, ZHAO-XIA WANG ${ }^{2}$ and ZHEN-YING LIU ${ }^{1}$ \\ Departments of ${ }^{1}$ Neurology and ${ }^{2}$ Gerontology, Linyi People's Hospital, Linyi, Shandong 276003, P.R. China
}

Received January 15, 2016; Accepted March 9, 2017

DOI: $10.3892 / \mathrm{mmr} .2017 .6610$

\begin{abstract}
R-1271 is a multifunctional post-translational modulator, which is involved in several diseases. However, the association between microRNA (miR)-1271 and fibronectin 1 (FN1) remains to be fully elucidated in neuroglioma. In the present study, it was hypothesized that a post-translational mechanism of miR-1271 regulates the expression of FN1 in the progression of neuroglioma. The present study aimed to investigate the clinical significance and underlying molecular mechanisms of miRNA-1271 in the development of glioma. The miR-1271 levels in glioma tissues and cell lines were assessed using reverse transcription-quantitative polymerase chain reaction (RT-qPCR). miR-1271 mimics and inhibitors were transfected to gain or loss of miR-1271 function. Cell proliferation was analyzed by using an MTT assay. The targeted genes were predicted by a bioinformatics algorithm and confirmed by a dual luciferase reporter assay. The mRNA and protein levels were assessed by RT-qPCR and western blotting. The results showed that miR-1271 was downregulated in glioma tumor tissues and cell lines. In addition, it was demonstrated that low levels of miR-1271 in patients with glioma were correlated with low survival rate. In vitro, the cell viability was significantly suppressed following transfection with miRNA-1271 mimics and increased following transfection with the miRNA-1271 inhibitor. The miRNA-1271 mimics induced cell apoptosis and the miRNA-1271 inhibitor suppressed cell apoptosis in $\mathrm{H} 4$ and U251 cell lines. Furthermore, the 3'-untranslated region of FN1 was bound by miR-1271. Therefore, it was concluded that miR-1271 inhibited glioma cell growth by targeting FN1, and a low level of miR-1271 in glioma tumor tissues was associated with lower survival rates in patients with glioma.
\end{abstract}

Correspondence to: Dr Zhen-Ying Liu, Department of Neurology, Linyi People's Hospital, 27 Jiefang Road, Linyi, Shandong 276003, P.R. China

E-mail:1iu_zyh09@163.com

Key words: microRNA-1271, fibronectin 1, neuroglioma

\section{Introduction}

Neuroglioma is the most common primary malignant tumor of the adult central nervous system and is known for its aggressive proliferation $(1,2)$. Despite advances in the diagnosis and therapeutic strategies for neuroglioma, the average survival rate of patients with malignant glioma has improved only marginally in previous decades, and $>70 \%$ of patients succumb to the mortality within 2 years of diagnosis (3-5). Currently, the treatment of neuroglioma primarily includes surgery, radiotherapy and chemotherapy, however, there are a number of side effects and tangible improvements in the final clinical outcome of patients are minimal (6). In order to improve clinical outcomes, understanding of the exact molecular mechanisms in the development and progression of neuroglioma is considered essential. Previous studies have demonstrated that epidermal growth factor receptor, vascular endothelial growth factor, the Akt-pathway and the nuclear factor- $\mathrm{\kappa B}$ pathway are associated with glioma invasion (7). However, the underlying molecular mechanisms of microRNA (miRNA)-mediated post-transcriptional regulation in glioma remain to be elucidated.

miRNAs are a class of small non-coding (18-25 nucleotides), naturally occurring endogenous, RNA molecules, which regulate the translation of messenger RNAs (mRNAs) by binding to the 3'-untranslated regions (3'-UTRs) of target mRNAs (8). The key features of miRNAs involve their regulation of cell proliferation, differentiation and apoptosis in various cell types $(9,10)$. An increasing number of studies have demonstrated that the pathogenic changes in various tissues are linked to miRNAs, and abnormal miRNAs expression via various cell signaling pathways regulates cancer development (11). A number of miRNAs have been identified to be markedly upregulated or downregulated in neuroglioma $(3,7,12,13)$. A previous study confirmed that low levels of miR-1271 in cancer tissues are correlated with a low rate of patient survival, and the overexpression of miR-1271 can inhibit the proliferation of cancer cells, and post-transcriptional regulatory mechanisms via targeting of cyclin G1 (14), forkhead box Q1 (15) or homeobox A5 (HOXA5) by miR-1271 have been reported in various types of cancer (16). However, the molecular mechanisms underlying the involvement of miR-1271 in glioma by targeting fibronectin 1 (FN1) remain to be elucidated.

FN1 is an extracellular matrix (ECM) glycoprotein, which is present in blood plasma in its soluble form (plasma type) and 
in its insoluble form (cellular type) as a component of the ECM in almost every tissue within organisms (17). Previous studies have indicated that overexpressed $\mathrm{FN}$ distorts the architecture of the liver, and leads to hepatic cirrhosis and consequently to hepatocellular cancer $(18,19)$. The abundant ECM in the glioma microenvironment is critical in the maintenance of glioma morphology, cell differentiation and proliferation, and FNs are expressed at high levels in the ECM of glioma spheroids in vitro and glioma tissues in vivo (20). However, the feasibility of post-transcriptional regulation via FN1 as a therapeutic target for glioma therapy remains to be elucidated. The present study investigated whether the expression of FN1 was regulated by miRNAs, and whether the tumorigenic role of FN1 was affected by miRNAs in vitro.

\section{Materials and methods}

Patient samples. A total of 36 pairs of glioma tumor tissues and corresponding adjacent normal tissues (NC) were collected from patients who had undergone surgical excision at Linyi People's Hospital (Linyi, China) between June 2010 and June 2014. All collected tissue samples were immediately stored in liquid nitrogen until use. The human tissue samples were obtained with written informed consent from all patients. The present study was approved by the Ethics Committee of the Linyi People's Hospital. The clinicopathological characteristics of the patients are shown in Table I.

Cell culture. Glioma cell lines (H4, A172, U251 and U87-MG) and a normal astrocyte cell line (HA-1800) were cultured in Dulbecco's modified Eagle's medium (Gibco; Thermo Fisher Scientific, Inc., Waltham, MA, USA) containing $10 \%$ fetal calf serum (Gibco; Thermo Fisher Scientific, Inc.), $10 \%$ L-glutamine, $0.5 \%$ penicillin/streptomycin, $10 \%$ nonessential amino acids and $10 \%$ pyruvate, in a $5 \% \mathrm{CO}_{2}$ atmosphere at $37^{\circ} \mathrm{C}$.

Analysis of proliferation using a 3-(4,5-dimethylthiazol-2-yl)-2,5-diphenyltetrazolium bromide (MTT) assay. Cell $\left(2 \times 10^{5}\right.$ cells/well) proliferation was measured using an MTT Cell Proliferation/Viability Assay kit (R\&D Systems, Inc., Minneapolis, MN, USA) according to the manufacturer's protocol.

Caspase-3 activity assay. Cells $\left(2 \times 10^{6}\right.$ cells/well) lysates were prepared in NP-40 buffer at ice-bath condition for $15 \mathrm{~min}$ and centrifuged at $900 \times \mathrm{g}$ for $10 \mathrm{~min}$ at $4^{\circ} \mathrm{C}$, and the supernatant was collected. In brief, $20 \mu \mathrm{l}$ of cell lysate incubated with anti-caspase-3 antibody (cat. no. sc-7272; dilution, $1: 200)$ at $37^{\circ} \mathrm{C}$ for $1 \mathrm{~h}$. The immunocomplexes were then incubated with peptide substrate $(2 \mu \mathrm{l}$ of $10 \mathrm{mM}$ acetyl-Asp-Glu-Val-Asp-p-nitroanilide) in assay buffer (100 mM Hepes, pH 7.5, 20\% v/v glycerol, 5 mM dithiothreitol, and $0.5 \mathrm{mM}$ EDTA) for $2 \mathrm{~h}$ at $37^{\circ} \mathrm{C}$. The release of p-nitroaniline was measured at $405 \mathrm{~nm}$ using an ELISA reader (MD SpectraMax M5; Molecular Devices, LLC, Sunnyvale, CA, USA) according to the manufacturer's protocol.

Quantification of apoptosis using flow cytometry. The quantitative assessment of apoptotic cells was assessed using the terminal deoxynucleotidyl transferase-mediated deoxyuridine triphosphate nick end labeling (TUNEL) method, which enabled examination of DNA-strand breaks during apoptosis using a BD ApoAlertTM DNA Fragmentation Assay kit (BD Biosciences, Franklin Lakes, NJ, USA). The cells were trypsinized, fixed with $4 \%$ paraformaldehyde and permeabilized with $0.1 \%$ Triton-X-100 in $0.1 \%$ sodium citrate. Subsequent to being washed with PBS three times, the cells $\left(2 \times 10^{5}\right.$ cells/well $)$ were incubated with the reaction mixture for $60 \mathrm{~min}$ at $37^{\circ} \mathrm{C}$. The cells were immediately analyzed using a FACScan flow cytometer and the CellQuest version 5.1 (BD Biosciences).

Immunohistochemical staining. The paraffin-embedded tumor tissues were cut into $\sim 4 \mu \mathrm{m}$ sections and mounted on glass slides for staining with immunoperoxidase. The paraffinized sections were heated in an oven at $65^{\circ} \mathrm{C}$ for $24 \mathrm{~h}$, dewaxed to water and rinsed with PBS three times. The washed sections were placed in EDTA buffer for microwave antigen retrieval, boiled, and then boiled at a low heat following an interval of $10 \mathrm{~min}$. Following natural cooling, the sections were washed with PBS three times, and were then placed into $3 \%$ hydrogen peroxide solution for incubation at room temperature for $10 \mathrm{~min}$, to block endogenous peroxidase. The sections were washed with PBS three times, and $5 \%$ bovine serum albumin (BSA; cat. no. ST023; Beyotime Institute of Biotechnology, Haimen, China) was added for $20 \mathrm{~min}$ at room temperature. Following removal of the BSA, each section was incubated with $50 \mu$ l diluted FN1-antibody (cat. no. sc-81769; dilution, 1:100; Santa Cruz Biotechnology, Inc., Santa Cruz, CA, USA) overnight at $4^{\circ} \mathrm{C}$, and then washed with PBS three times. Following the removal of PBS, each section was incubated with 50-100 $\mu$ l secondary (cat. no. sc-516102; dilution, 1:2,000; Santa Cruz Biotechnology, Inc.) antibody at $4^{\circ} \mathrm{C}$ for $50 \mathrm{~min}$. The sections were washed again with PBS three times, and each section was added to 50-100 $\mu$ l freshly prepared DAB solution with the use of a microscope for controlling color. Following washing, the sections were counterstained with hematoxylin, rinsed with tap water, dehydrated and mounted for visualization under a microscope (Leica DM 2500; Leica Microsystems GmbH, Wetzlar, Germany).

Luciferase reporter gene activity assay. The 3'-UTR of the FN1 gene containing the predicated target sites for miRNA-1271 was obtained by online predict software (miRanda-mirSVR; www. microrna.org), DIANA TOOLS (diana.imis.athena-innovation. gr) and TargetScan (www.targetscan.org), and synthesized by GenePharma Co., Ltd. (Shanghai, China). The fragment was inserted into multiple cloning sites in the pMIR-REPORT luciferase microRNA expression reporter vector (Ambion; Thermo Fisher Scientific, Inc.). The H4 cells (5x105 cells/well) were co-transfected with luciferase reporters containing the FN1 3'-UTR and miRNA-1271 mimics (50 or $100 \mathrm{nM}$ ) using Lipofectamine 2000 (Invitrogen; Thermo Fisher Scientific, Inc.). The luciferase activity was measured using a dual luciferase reporter assay kit (cat. no. RG027; Beyotime Institute of Biotechnology) according to the manufacturer's protocol.

Transfection with miRNA-1271 mimics and inhibitor. The FAM modified 2'-OMe-oligonucleotides were chemically synthesized and purified using high-performance 
Table I. Correlation between clinicopathological factors and expression levels of miRNA-1271 in patients with glioma.

\begin{tabular}{|c|c|c|c|c|}
\hline Variable & Patients (n) & miRNA-1271 low (n) & miRNA-1271 high (n) & P-value \\
\hline Sex & & & & 0.385 \\
\hline Male & 20 & 9 & 11 & \\
\hline Female & 16 & 9 & 7 & \\
\hline Age (years) & & & & 0.517 \\
\hline$<60$ & 22 & 10 & 12 & \\
\hline$\geqq 60$ & 14 & 8 & 6 & \\
\hline \multicolumn{5}{|c|}{ Tumor size (cm) } \\
\hline$<3$ & 12 & 7 & 5 & 0.274 \\
\hline$\geq 3$ & 24 & 11 & 13 & \\
\hline TNM stage & & & & 0.001 \\
\hline I-II & 22 & 14 & 8 & \\
\hline III-IV & 14 & 4 & 10 & \\
\hline
\end{tabular}

miRNA, microRNA; TNM, tumor-node-metastasis.

liquid chromatography (GenePharma, Co., Ltd.). The 2'-OMe-miR-1271 mimics were composed of RNA duplexes with the following sequence: 5'-CUUGGCACCUAGCAA GCACUCA-3'. The sequences of the 2'-OMe-miR-1271 inhibitor and 2'-Ome-scramble oligonucleotides were as follows: 5'-UGAGUGCUUGCUAGGUGCCAAG-3' and 5'-UCAGGA GCGUUGCCUGGCUCGG-3'. The H4 (5x10 5 cells/well) and U251 cells $\left(5 \times 10^{5}\right.$ cells/well $)$ were transfected using Lipofectamine 2000 (Invitrogen; Thermo Fisher Scientific, Inc.) at a final concentration of $100 \mathrm{nM}$. At $24 \mathrm{~h}$ post-transfection, the culture medium was replaced and, cells were harvested at $48 \mathrm{~h}$ for analysis.

Reverse transcription-quantitative PCR (RT-qPCR) analysis. RNA extraction was performed using TRIzol according to the manufacturer's protocol (Invitrogen; Thermo Fisher Scientific, Inc.). The synthesis of cDNAs was performed by RT reactions with $4 \mu \mathrm{g}$ of total RNA using moloney murine leukemia virus reverse transcriptase (Invitrogen; Thermo Fisher Scientific, Inc.) with oligo dT (15) primers (Fermentas; Thermo Fisher Scientific, Inc.) according to the manufacturer's protocol. PCR reaction mixtures $(25 \mu \mathrm{l})$ were prepared, including $12.5 \mu \mathrm{l}$ SYBR-Green Supermix (Bio-Rad Laboratories, Inc., Hercules, CA, USA), $1 \mu \mathrm{l}$ cDNA, $300 \mathrm{nM}$ of each primer, and DEPC $\mathrm{H}_{2} \mathrm{O}$ to a final volume of $25 \mu \mathrm{l}$. The levels of miRNA-1271 were quantified using the mirVana qRT-PCR miRNA detection kit (Ambion; Thermo Fisher Scientific, Inc.) in conjunction with qPCR with SYBR Green. Following the cycling reactions: $95^{\circ} \mathrm{C}$ for $10 \mathrm{~min}$, followed by 40 cycles of $95^{\circ} \mathrm{C}$ for $15 \mathrm{sec}, 58^{\circ} \mathrm{C}$ for $30 \mathrm{sec}$, and $72^{\circ} \mathrm{C}$ for $30 \mathrm{sec}$, the quantification cycle $(\mathrm{Cq})$ was determined and the relative miRNA-1271 level was calculated based on the $2^{-\Delta \Delta \mathrm{Cq}}$ method (21) and normalized to the level of U6 in each sample, and relative expression levels of FN1 were normalized to GAPDH. PCR was performed using the following primers: FN1, forward 5'-GCGCCGGCTGTGCTG CACAGG-3' and reverse 5'-GCCTGGGGACAGCGGTGC CC-3'; GAPDH, forward 5'-GCACCGTCAAGCTGAGAAC-3' and reverse 5'-TGGTGAAGACGCCAGTGGA-3'.
Western blot analysis. The tumor tissues, corresponding adjacent normal tissues and glioma cells were homogenized and extracted in NP-40 buffer, respectively, followed by 5-10 min boiling and centrifugation at $12,000 \mathrm{x} \mathrm{g}$ for $15 \mathrm{~min}$ at $4^{\circ} \mathrm{C}$ to obtain the supernatant. Protein concentrations were determined using the bicinchoninic acid kit (cat. no. BCA1-1KT; Sigma-Aldrich; Merck KGaA, Darmstadt, Germany). Samples containing $50 \mu \mathrm{g}$ of protein were separated on a $10 \%$ SDS-PAGE gel and transferred onto nitrocellulose membranes (Bio-Rad Laboratories, Inc.). Following saturation with 5\% (w/v) non-fat dry milk in TBS and $0.1 \%$ (w/v) Tween-20 (TBST), the membranes were incubated at $37^{\circ} \mathrm{C}$ for $2 \mathrm{~h}$ with the following primary antibodies: FN1 (cat. no. sc-81769; 1:1,000), caspase-3 (cat. no. sc-271028; 1:2,000), B-cell lymphoma-2 (Bcl-2; cat. no. sc-56015; 1:1,000) and Bcl-2-associated X protein (Bax; cat. no. sc6236; 1:1,000) from Santa Cruz Biotechnology, Inc. Following three washes with TBST, the membranes were incubated with the appropriate horseradish peroxidase-conjugated secondary antibody (cat. no. sc-516102; 1:10,000) for $1 \mathrm{~h}$ at $37^{\circ} \mathrm{C}$, following visualized using chemiluminescence (Thermo Fisher Scientific, Inc.). Densitometry was used to assess the signals with Quantity One software version 4.5 (Bio Rad Laboratories, Inc., Hercules, CA, USA) and normalized to $\beta$-actin (cat. no. sc-130065; 1:2,000).

Statistical analysis. The data from experiments are reported as the mean \pm standard deviation for each group. All statistical analyses were performed using PRISM version 5.0 (GraphPad Software, Inc., La Jolla, CA, USA). Statistical differences between two groups were determined using Student's t test. The correlation between FN1 and miR-1271 levels was analyzed using linear regression analysis. Groups were compared using one-way analysis of variance, followed by Tukey's multiple comparison as a post hoc test to compare the mean values of each group. Survival rates were calculated using the Kaplan-Meier method with the log-rank test applied for comparison. Differences between the expression levels of miRNA-1271 and different clinicopathological factors were 

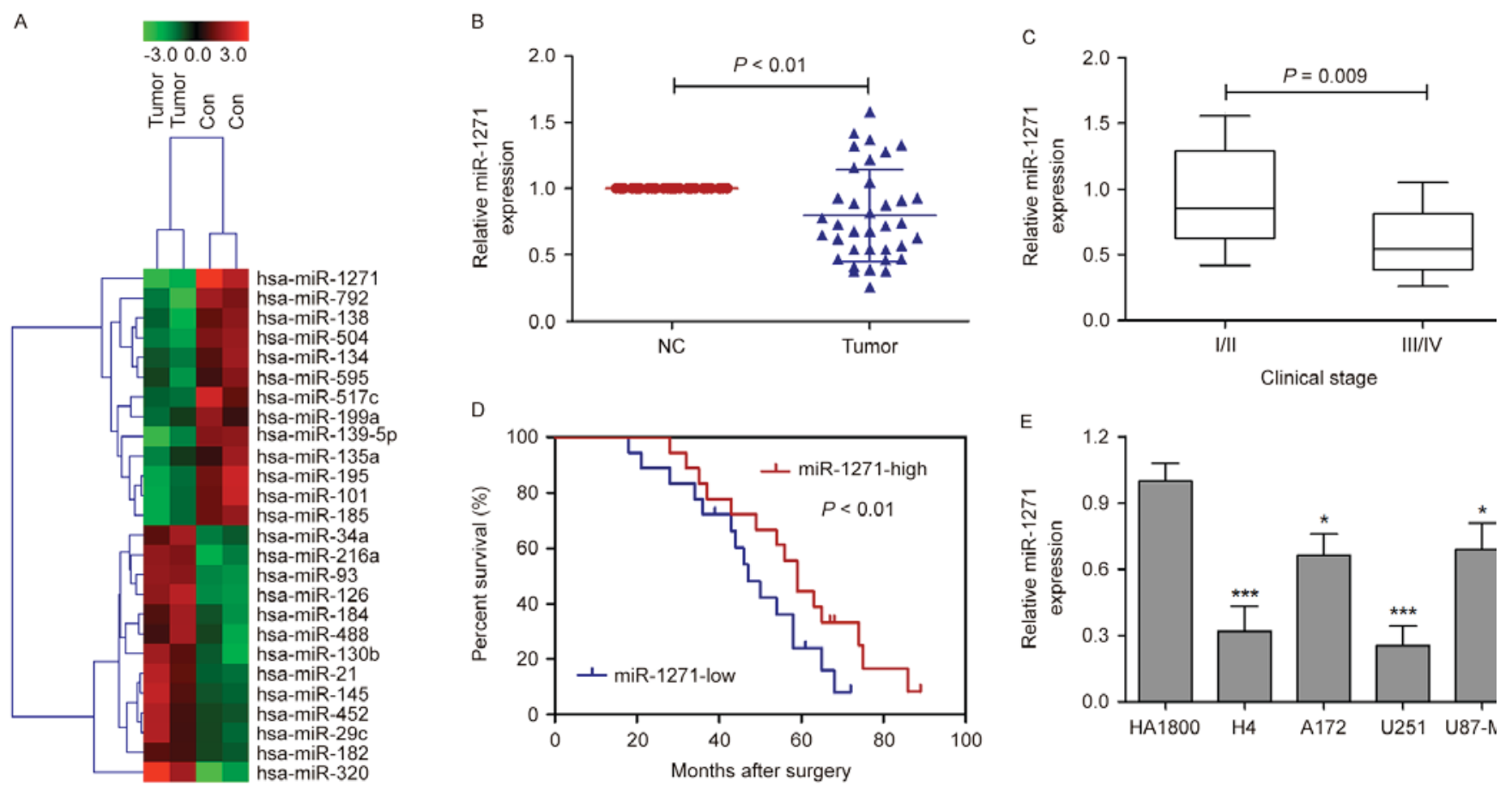

Figure 1. Profiles of miRNAs in glioma tissues and corresponding adjacent normal tissues. (A) Microarray and hierarchical cluster analysis of the significantly regulated miRNAs in patients with glioma. MeV software version 4.2.6 was used for representation of data. Correlation similarity matrix and average linkage algorithms were used in the cluster analysis. Each row represents an individual miRNA and each column represents a sample. The dendrogram at the left and above display similarities in expression among miRNAs and samples individually. The color legend at the top represents the expression level of miRNA, with red indicating high expression and green indicating low expression. (B) miRNA-1271 expression was examined using reverse transcription-quantitative polymerase chain reaction analysis and normalized to the expression of U6 in 36 pairs of glioma tumor tissues (tumor) and corresponding adjacent normal tissues. (C) Relative miRNA-1271 expression was measured in different clinical stages. (D) A Kaplan-Meier survival curve was used to evaluate whether expression levels of miRNA-1271 were associated with overall survival rate. (E) miRNA-1271 expression was measured in H4, A172, U251 and U87-MG glioma cell lines, and the normal astrocyte HA-1800 cell line. Values were expressed as the mean \pm standard deviation. ${ }^{*} \mathrm{P}<0.05$ and ${ }^{* * *} \mathrm{P}<0.001$, vs. HA-1800 group. miRNA, microRNA; NC, normal tissues; Con, control.

calculated using the $\chi^{2}$ test. $\mathrm{P}<0.05$ was considered to indicate a statistically significant difference.

\section{Results}

Profiles of miRNAs in glioma tissues and corresponding adjacent normal tissues. To identify the critical miRNAs involved in glioma, tumor tissues and corresponding adjacent normal tissues were collected from 36 patients with neuroglioma, and miRNA microarray chips were performed in two pairs of randomly selected tumor tissues and corresponding adjacent normal tissues. A total of 13 miRNAs (miR-134, miR-1271, miR-139-5p, miR-517c, miR-195, miR-135a, miR-101, miR-199a, miR-792, miR-138, miR-504, miR-595 and miR-185) were downregulated and 13 miRNAs were upregulated in the tumor tissues, compared with corresponding adjacent normal tissues (Fig. 1A). Among these miRNAs, the levels of miRNA-1271 were markedly lower, compared with those of other miRNAs in the tumor tissues. Therefore, the present study focused on miRNA-1271. To further validate the miRNA microarray results, the levels of miRNA-1271 were measured using RT-qPCR analysis in the 36 pairs of tumor tissues and corresponding adjacent normal tissues. The results showed that the expression of miRNA-1271 was markedly suppressed in tumor tissues, compared with corresponding adjacent normal tissues (Fig. 1B). In addition, to validate the clinical significance of miRNA-1271 in glioma, the association between the expression of miRNA-1271 and clinicopathological parameters, including tumor size and clinical stage, were assessed. As shown in Fig. 1C, the expression levels of miRNA-1271 were significantly lower in patients with stage III/IV glioma, compared with patients with stage I/II glioma, however, no significant difference was found in the expression of miRNA-1271 with tumor size in the patients with glioma (data not shown). A Kaplan-Meier survival curve was used to evaluate whether the expression level of miRNA-1271 was associated with overall survival rate. Patients were segregated into a miRNA-1271-high group and miRNA-1271-low group according to the median expression of miRNA-1271 in tumor tissues. Patients with low levels of miRNA-1271 had a significantly poorer prognosis, compared with those with high expression (Fig. 1D; P<0.01). Based on the above observations, analysis of the expression of miRNA-1271 was performed among four glioma cell lines (H4, A172, U251 and U87-MG) and a normal astrocyte cell line (HA-1800). miRNA-1271 levels were significantly downregulated in the four glioma cell lines, compared with the level in the HA-1800 cells, particularly in the H4 and U251 cell lines (Fig. 1E). Therefore, the H4 and U251 cell lines were selected as representative glioma cells in the following experiments.

Expression of FN1 is upregulated in glioma tissues. The results of the present study showed that the expression of FN1 differed significantly between the glioma tissues and the 


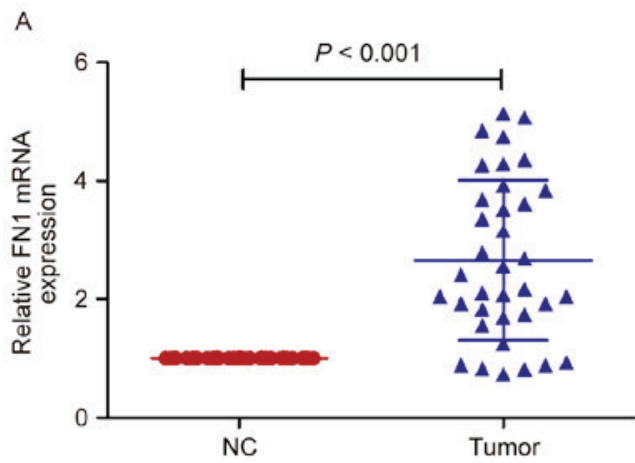

B

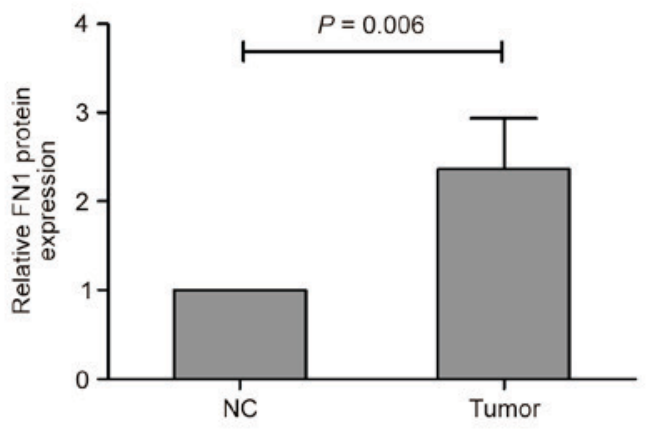

C

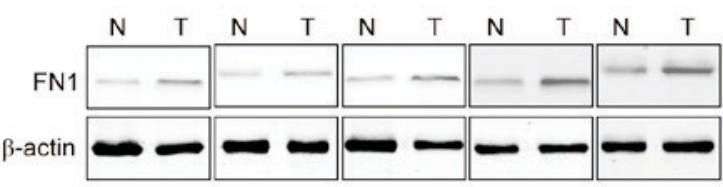

Tumor
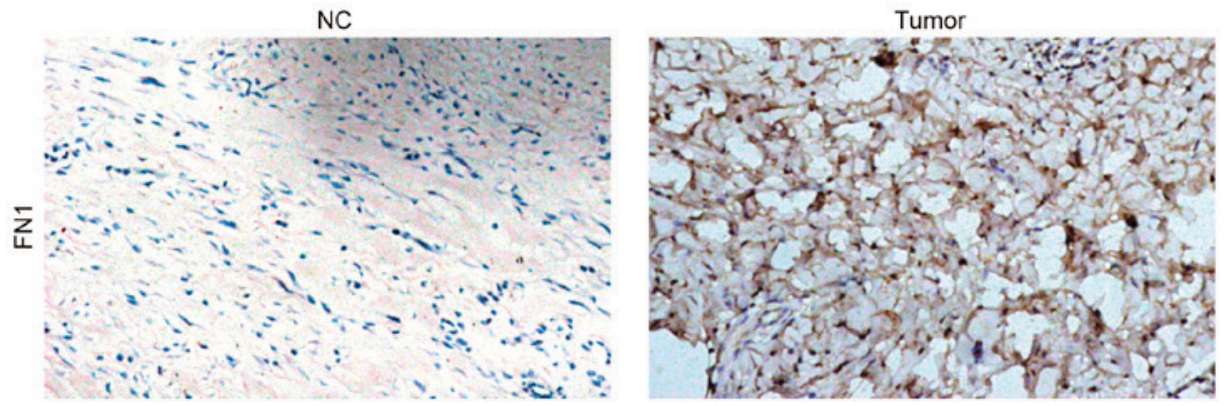

$\mathrm{E}$

D
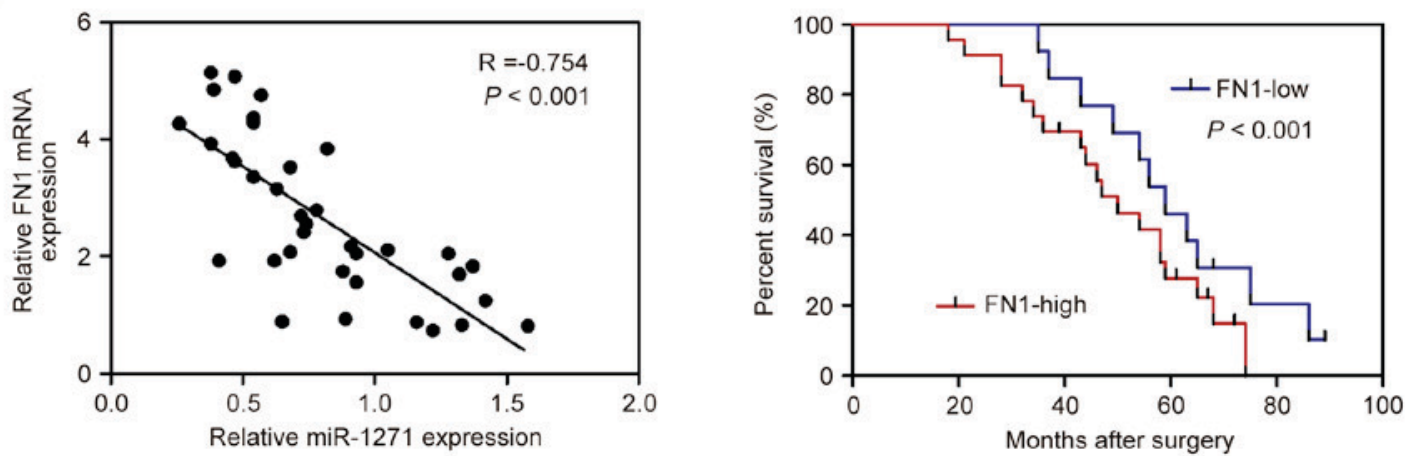

Figure 2. Expression of FN1 is upregulated in glioma tissues. (A) mRNA and (B) protein expression levels of FN1 were measured using reverse transcription-quantitative polymerase chain reaction and western blot analysis, respectively. (C) Expression of FN was measured using immunohistochemical staining in glioma tumor tissues (tumor) and corresponding adjacent normal tissues (magnification, x100). (D) Inverse correlation of miRNA-1271 and mRNA expression of FN1 in glioma tumor tissues. (E) A Kaplan-Meier survival curve was used to evaluate whether mRNA expression levels of FN1 were associated with overall survival rate in patients with glioma. FN1, fibronectin 1; miR, microRNA; T, tumor; N/NC, normal non-tumor tissues.

corresponding adjacent normal tissues, and the mRNA levels of FN1 were significantly increased in the glioma tissues, compared with the corresponding adjacent normal tissues (Fig. 2A). In addition, the results of the western blot analysis (Fig. 2B) and immunohistochemical staining (Fig. 2C) showed a marked increase in the levels of FN1 in the glioma tissues, compared with those in the corresponding adjacent normal tissues. Spearman's rank correlation analysis showed that the expression levels of FN1 and miRNA-1271 were inversely correlated in the 36 human glioma tissues (Spearman's $\mathrm{R}=-0.754$; $\mathrm{P}<0.001$; Fig. 2D). Furthermore, a Kaplan-Meier survival curve was used to evaluate whether the expression level of FN1 was associated with overall survival rate. Patients were segregated into an FN1-high group and FN1-low group according to the median mRNA expression of FN1 in tumor tissues. Patients with high mRNA levels of FN1 had a significantly poorer prognosis, compared with those with a low levels (Fig. 2E; $\mathrm{P}<0.001$ ).

FN1 is a direct target of miRNA-1271. To investigate the mechanisms by which miRNA-1271 regulates the expression of FN1. Binding sites in the FN1 3'-UTR were predicted using bioinformatics analysis (Fig. 3A). The pmirGLO-wild-FN1 and pmirGLO-mut-FN1 plasmids were synthesized and the FN1 

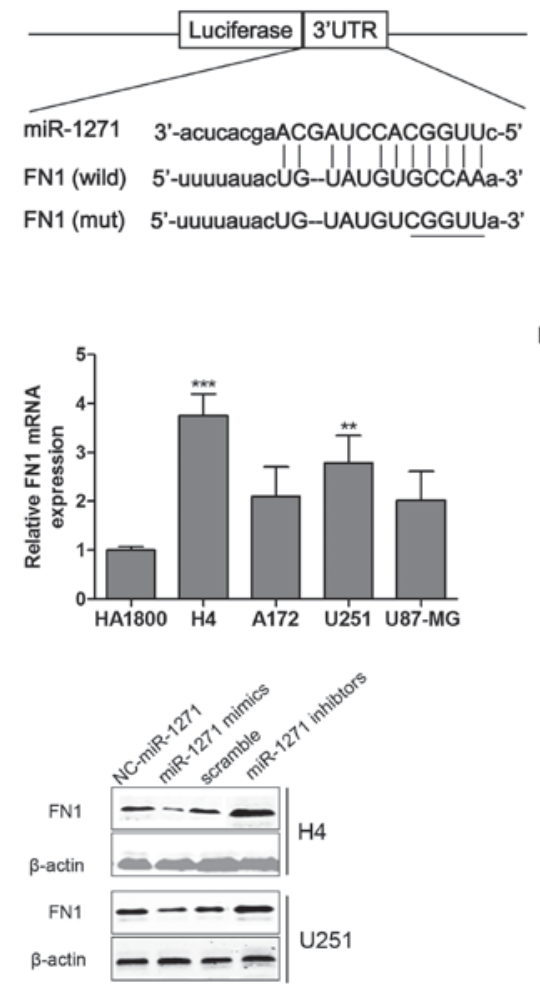
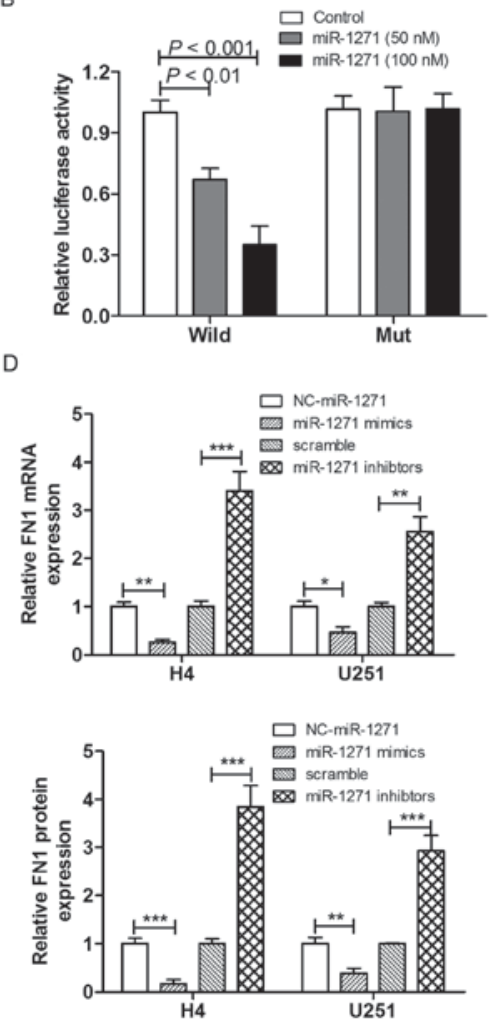

Figure 3. FN1 is a direct target of miRNA-1271. (A) Schematic representation of the putative miRNA-1271 binding site in the FN1 3'UTR and (B) luciferase activity assay. (C) mRNA expression levels of FN1 were analyzed using RT-qPCR analysis in H4, A172, U251 and U87-MG glioma cell lines and the HA-1800 normal astrocyte cell line. (D) mRNA and (E) protein expression levels of FN1 were analyzed using RT-qPCR or western blot analysis, respectively, in H4 and U251 cell lines transfected with miRNA-1271 mimics or inhibitor. Values were expressed as the mean \pm standard deviation ( $\mathrm{n}=3$ in each group). ${ }^{* *} \mathrm{P}<0.01$ and ${ }^{* * *} \mathrm{P}<0.001$, vs. control group. FN1, fibronectin 1; miR, microRNA; RT-qPCR, reverse transcription-quantitative polymerase chain reaction; NC, normal tissue; 3'UTR, 3'untranslated region.

3'-UTR luciferase reporter assay was performed in H4 cells. The luciferase activity was markedly decreased in $\mathrm{H} 4$ cells transfected with the miRNA-1271 mimics (50 or $100 \mathrm{nM}$ ) and pmirGLO-wild-FN1, however, no significant difference in luciferase activity was observed between $\mathrm{H} 4$ cells transfected with the pmirGLO-mut-FN1 and the control group (Fig. 3B). These results suggested that FN1 was a direct target of miRNA-1271. Based on the above, the mRNA expression levels of FN1 were measured among the four glioma cell lines (H4, A172, U251 and U87-MG) and the normal astrocyte line (HA-1800). The results indicated that FN1 was significantly upregulated in the H4 and U251 cell lines, compared with the HA-1800 cell lines, however, there was no significant difference in the mRNA expression of FN1 in the A172 and U87-MG cells, compared with the HA-1800 cells (Fig. 3C). To confirm the association between FN1 and miRNA-1271, RT-qPCR and western blot analyses were performed to investigate the expression levels of FN1 in the H4 and U251 cell lines following transfection with miRNA-1271 mimics, miRNA-1271 inhibitor, or the corresponding NC or scramble. As shown in Fig. 3D and E, the mRNA and protein expression levels of FN1 in the $\mathrm{H} 4$ and U251 cell lines were significantly decreased following transfection with the miRNA-1271 mimics and increased following transfection with the miRNA-1271 inhibitor.

Overexpression of miRNA-1271 induces cell apoptosis. As described above, the present study found that the expression of
miRNA-1271 was downregulated in tumor tissues and glioma cell lines. It was also demonstrated that miRNA-1271 directly regulated the expression of FN1 via binding sites within the 3'-UTR of FN1. To examine the role of miRNA-1271 in glioma cell apoptosis in vitro, cell viability was examined using an MTT assay in cells transfected with the miRNA-1271 mimics or inhibitor for 24,48 and $72 \mathrm{~h}$. The viabilities of the $\mathrm{H} 4$ and U251 cells were significantly suppressed following transfection with the miRNA-1271 mimics and increased following transfection with the miRNA-1271 inhibitor (Fig. 4A). An apoptosis assay, caspase-3 activity assay and TUNEL staining were performed following transfection of the $\mathrm{H} 4$ and $\mathrm{U} 251$ cells with miRNA-1271 mimics or inhibitor for $48 \mathrm{~h}$. The results indicated that the activity of caspase-3 in the miRNA-1271 mimic group was significantly higher, compared with that of the control group. This was in contrast to the $\mathrm{H} 4$ and $\mathrm{U} 251$ cells transfected with miRNA-1271 inhibitor, which suppressed the activity of caspase-3 (Fig. 4B). The TUNEL staining showed that the miRNA-1271 mimics induced apoptosis, whereas the miRNA-1271 inhibitor suppressed apoptosis of the $\mathrm{H} 4$ and U251 cells (Fig. 4C). Furthermore, apoptosis-associated proteins were measured using western blot analysis in $\mathrm{H} 4$ and U251 cells transfected with miRNA-1271 mimics or inhibitor for $48 \mathrm{~h}$. As shown in Fig. 4D-F, the protein expression levels of caspase-3 and Bax were markedly increased following transfection with the miRNA-1271 mimics and decreased following transfection with the miRNA-1271 inhibitor. By contrast, the protein 
A
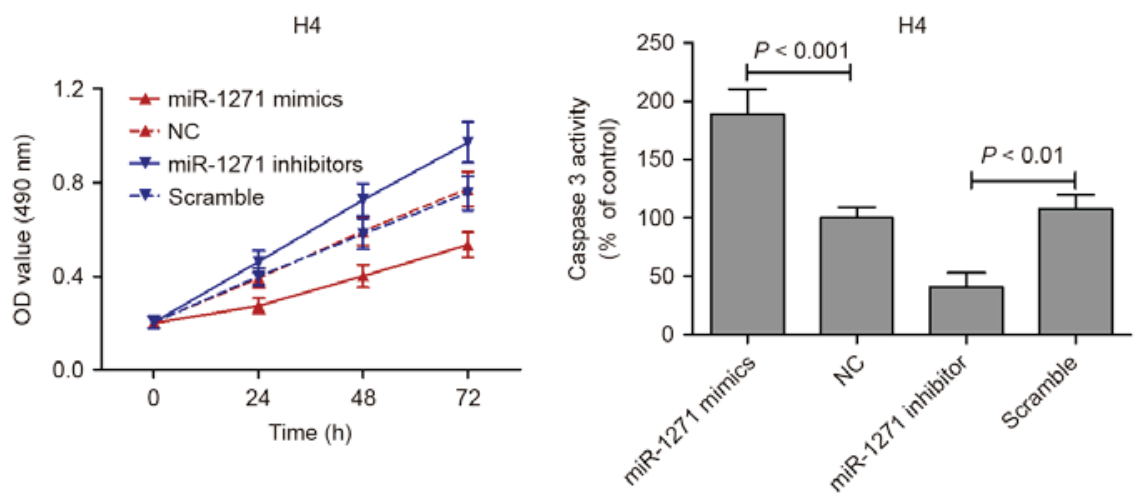

U251
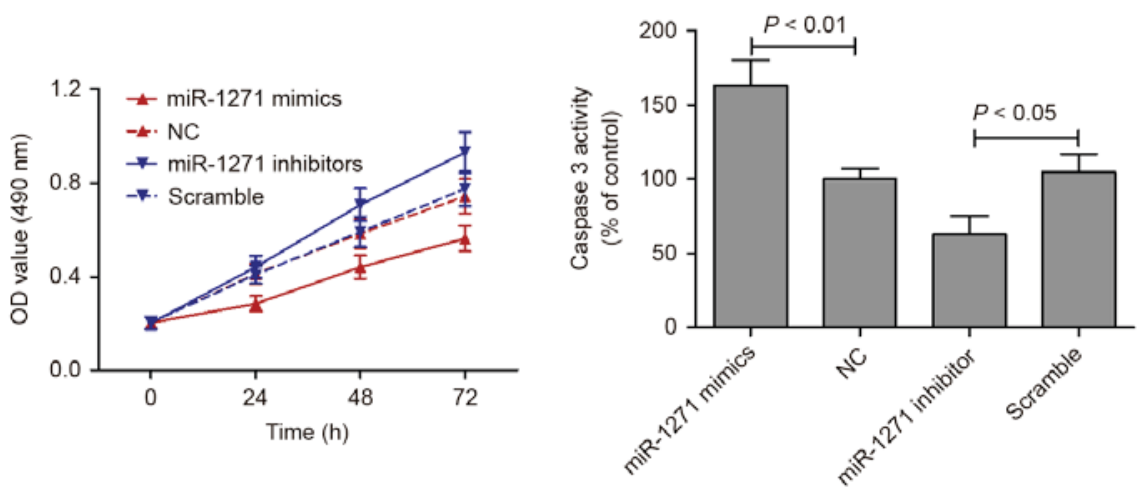

$E$

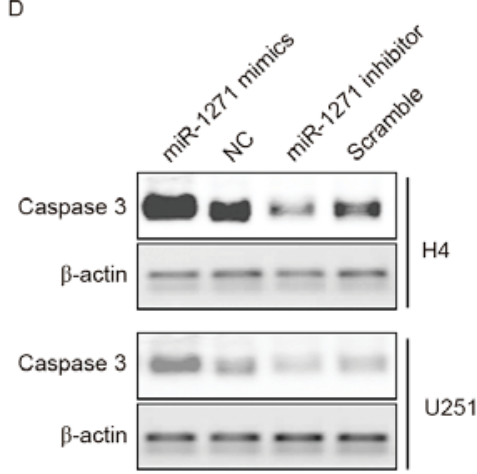

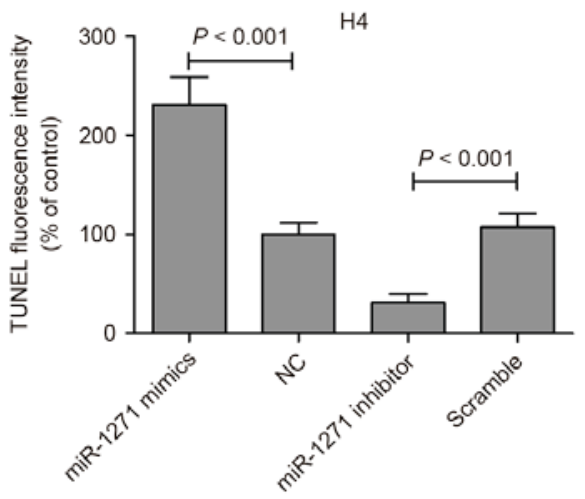

U251
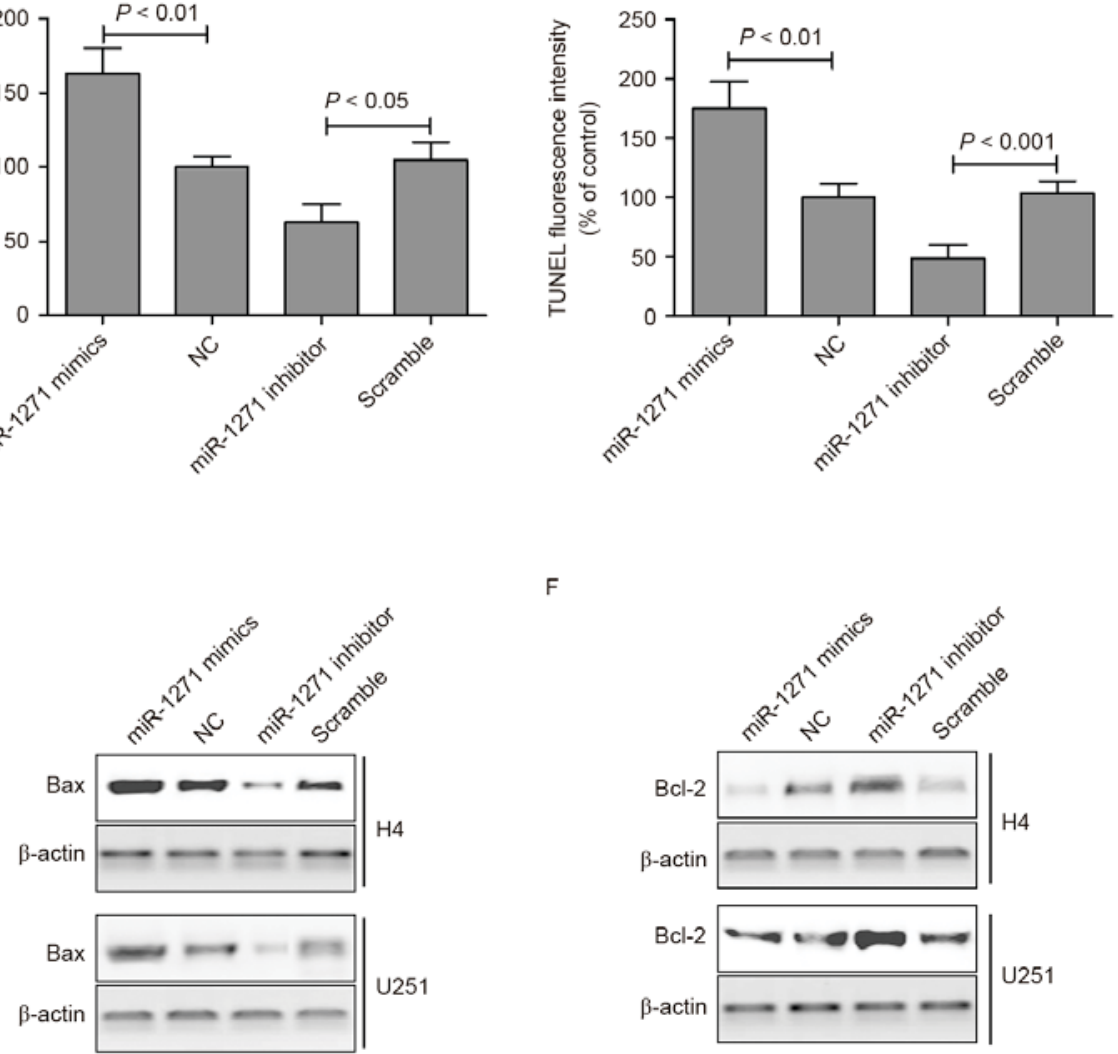

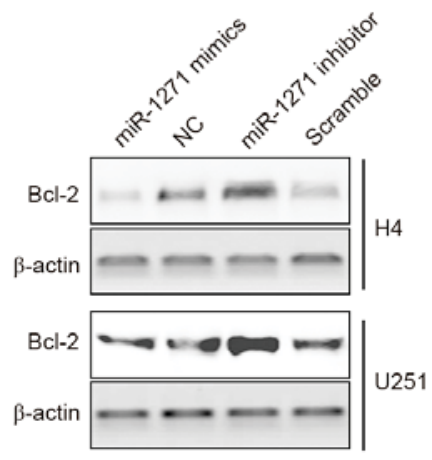

Figure 4. Overexpression of miRNA-1271 induces cell apoptosis. (A) 3-(4,5-dimethylthiazol-2-yl)-2,5-diphenyltetrazolium bromide assays were performed on $\mathrm{H} 4$ and U251 cell lines transfected with miRNA-1271 mimics or inhibitor for 24, 48 and $72 \mathrm{~h}$. (B) A Caspase-3 activity assay and (C) TUNEL staining were performed following transfection of the $\mathrm{H} 4$ and $\mathrm{U} 251$ cell lines with miRNA-1271 mimics or inhibitor for $48 \mathrm{~h}$. The protein expression of (D) caspase-3, (E) Bax and (F) Bcl-2 were measured using western blot analysis in H4 and U251 cell lines transfected with miRNA-1271 mimics or inhibitor for 48 h. FN1, fibronectin 1; miR, microRNA; Bcl-2, B cell lymphoma-2; Bax, Bcl-2-associated X protein; NC, normal tissue; TUNEL, triphosphate nick-end labeling; OD, optical density.

expression of $\mathrm{Bcl}-2$ was significantly decreased following transfection with the miRNA-1271 mimics and increased following transfection with the miRNA-1271 inhibitor.

\section{Discussion}

Previous studies have demonstrated the essential role of miRNAs in the progression of cancer $(22,23)$, however, the role of miRNA-1271 in glioma has not been determined. In the present study, the clinical significance and underlying molecular mechanisms of miRNA-1271 in the development of glioma were investigated. The results showed that miRNA-1271 was downregulated in glioma tumor tissues and cell lines. In addition, it was demonstrated that low levels of miRNA-1271 in patients with glioma were correlated with low survival rates. In vitro, cell viability was significantly suppressed following transfection with the miRNA-1271 mimics and increased following transfection with the miRNA-1271 inhibitor. The miRNA-1271 mimics induced cell apoptosis, whereas the miRNA-1271 inhibitor suppressed cell apoptosis in the H4 
and U251 cell lines. Furthermore, 3'-UTR of FN1 was bound by miRNA-1271. Therefore, it was concluded that miRNA-1271 inhibited glioma cell growth by targeting FN1, and the levels of miRNA-1271 in glioma tumor tissues were associated with lower survival rates in patients with glioma.

Although it has been reported that miRNA-1271 is markedly suppressed in various types of tumor, and is associated with tumor proliferation and metastasis $(14,15,24,25)$, data concerning miRNA-1271 in human glioma remains limited. The results of the present study revealed the antitumor role of miRNA-1271 in human glioma and showed that miRNA-1271 offered potential for use as a prognostic predictor. The present study demonstrated for the first time, to the best of our knowledge, that miRNA-1271 was downregulated in glioma tumor tissues, compared with corresponding adjacent normal tissues, and was closely associated with clinical stage. However, how miRNA-1271 contributes to the malignant behavior of glioma cells remains to be fully elucidated. Previous studies have indicated that miRNA-1271 inhibits ovarian cancer growth by targeting cyclin G1 (14), regulates cisplatin resistance of human gastric cancer cell lines by targeting insulin-like growth factor 1 receptor, insulin receptor substrate 1 , mammalian target of rapamycin and $\mathrm{Bcl}-2$ (26), and promotes non-small-cell lung cancer cell proliferation and invasion by targeting HOXA5 (16). In addition, the downregulation of miRNA-1271 has been associated with the concomitant upregulation of glypican-3, one of the most abnormally expressed genes contributing to liver carcinogenesis (27). In the present study, bioinformatics analysis was performed, and it was found that miRNA-1271 directly regulated the expression of FN by targeting its 3'-UTR in glioma cells. miRNA-1271 mimics induced apoptosis and miRNA-1271 inhibitor suppressed apoptosis in the H4 and $\mathrm{U} 251$ cell lines. In addition, the correlation between miRNA-1271 and FN1 showed that endogenous miRNA-1271 was negatively correlated with the mRNA levels of FN1 in glioma tissues. These data provided further evidence of a functional link between miRNA-1271 and FN1 in human glioma.

In conclusion, the present study identified miRNA-1271 as a novel tumor suppressor, which inhibited human glioma cell proliferation and induced cell apoptosis through the suppression of FN1. The findings that miRNA-1271 targeted FN1 as a post-translational mechanism provides novel insight into the underlying mechanism and therapeutic strategies for the treatment of human glioma.

\section{References}

1. Tong YQ, Liu B, Zheng HY, Gu J, Liu H, Li F, Tan BH, Hartman M, Song C and Li Y: MiR-215, an activator of the CTNNBIP1/ $\beta$-catenin pathway, is a marker of poor prognosis in human glioma. Oncotarget 6: 25024-25033, 2015.

2. Shi R, Wang PY, Li XY, Chen JX, Li Y, Zhang XZ, Zhang CG, Jiang T, Li WB, Ding W and Cheng SJ: Exosomal levels of miRNA-21 from cerebrospinal fluids associated with poor prognosis and tumor recurrence of glioma patients. Oncotarget 6: 26971-26981, 2015.

3. Han L, Liu D, Li Z, Tian N, Han Z, Wang G, Fu Y, Guo Z, Zhu Z, Du $C$ and Tian Y: HOXB1 is a tumor suppressor gene regulated by miR-3175 in glioma. PloS One 10: e0142387, 2015.

4. Johnson DR and Galanis E: Incorporation of prognostic and predictive factors into glioma clinical trials. Curr Oncol Rep 15: 56-63, 2013.
5. Bageritz J, Puccio L, Piro RM, Hovestadt V, Phillips E, Pankert T, Lohr J, Herold-Mende C, Lichter P and Goidts V: Stem cell characteristics in glioblastoma are maintained by the ecto-nucleotidase E-NPP1. Cell Death Differ 21: 929-940, 2014.

6. Yang H and Wang Y: Five miRNAs considered as molecular targets for predicting neuroglioma. Tumour Biol 37: 1051-1059, 2016.

7. Fabbri E, Brognara E, Montagner G, Ghimenton C, Eccher A, Cantù $\mathrm{C}$, Khalil S, Bezzerri V, Provezza L and Bianchi N: Regulation of IL-8 gene expression in gliomas by microRNA miR-93. BMC Cancer 15: 661, 2015.

8. Paranjape T, Heneghan H, Lindner R, Keane FK, Hoffman A, Hollestelle A, Dorairaj J, Geyda K, Pelletier C, Nallur S, et al: A 3'-untranslated region KRAS variant and triple-negative breast cancer: A case-control and genetic analysis. Lancet Oncol 12: 377-386, 2011.

9. Fujii T, Shimada K, Tatsumi Y, Hatakeyama K, Obayashi C, Fujimoto K and Konishi N: microRNA-145 promotes differentiation in human urothelial carcinoma through down-regulation of syndecan-1. BMC Cancer 15: 818, 2015.

10. Hata A and Lieberman J: Dysregulation of microRNA biogenesis and gene silencing in cancer. Sci Signal 8: re3, 2015.

11. Kim M, Kasinski AL and Slack FJ: MicroRNA therapeutics in preclinical cancer models. Lancet Oncol 12: 319-321, 2011.

12. Li R, Li X, Ning S, Ye J, Han L, Kang C and Li X: Identification of a core miRNA-pathway regulatory network in glioma by therapeutically targeting miR-181d, miR-21, miR-23b, $\beta$-Catenin, CBP, and STAT3. PloS One 9: e101903, 2014.

13. Kouri FM, Ritner C and Stegh AH: miRNA-182 and the regulation of the glioblastoma phenotype-toward miRNA-based precision therapeutics. Cell Cycle 14: 3794-3800, 2015.

14. Liu X, Ma L, Rao Q, Mao Y, Xin Y, Xu H, Li C and Wang X: MiR-1271 inhibits ovarian cancer growth by targeting cyclin G1. Med Sci Monit 21: 3152-3158, 2015.

15. Xiang XJ, Deng J, Liu YW, Wan LY, Feng M, Chen J and Xiong JP: MiR-1271 inhibits cell proliferation, invasion and EMT in gastric cancer by targeting FOXQ1. Cell Physiol Biochem 36: 1382-1394, 2015.

16. Wang Y, Xu L and Jiang L: miR-1271 promotes non-small-cell lung cancer cell proliferation and invasion via targeting HOXA5. Biochem Biophys Res Commun 458: 714-719, 2015.

17. Liang CJ, Yen YH, Hung LY, Wang SH, Pu CM, Chien HF, Tsai JS, Lee CW, Yen FL and Chen YL: Thalidomide inhibits fibronectin production in TGF- $\beta 1$-treated normal and keloid fibroblasts via inhibition of the p38/Smad3 pathway. Biochem Pharmacol 85: 1594-1602, 2013.

18. Zeng C, Wang YL, Xie C, Sang Y, Li TJ, Zhang M, Wang R, Zhang Q, Zheng L and Zhuang SM: Identification of a novel TGF- $\beta$-miR-122-fibronectin $1 /$ serum response factor signaling cascade and its implication in hepatic fibrogenesis. Oncotarget 6: 12224-12233, 2015.

19. Hernandez-Gea V and Friedman SL: Pathogenesis of liver fibrosis. Annu Rev Pathol 6: 425-456, 2011.

20. Zhang B, Shen S, Liao Z, Shi W, Wang Y, Zhao J, Hu Y, Yang J, Chen J, Mei H, et al: Targeting fibronectins of glioma extracellular matrix by CLT1 peptide-conjugated nanoparticles. Biomaterials 35: 4088-4098, 2014.

21. Livak KJ and Schmittgen TD: Analysis of relative gene expression data using real-time quantitative PCR and the 2(-Delta Delta C(T)) method. Methods 25: 402-408, 2001.

22. Zhang L, Zhang S, Yao J, Lowery FJ, Zhang Q, Huang WC, Li P, Li M, Wang X, Zhang C, et al: Microenvironment-induced PTEN loss by exosomal microRNA primes brain metastasis outgrowth. Nature 527: 100-104, 2015.

23. Kontomanolis EN and Koukourakis MI: MicroRNA: The potential regulator of endometrial carcinogenesis. Microrna 4: 18-25, 2015.

24. Kong D, Zhang G, Ma H and Jiang G: miR-1271 inhibits OSCC cell growth and metastasis by targeting ALK. Neoplasma 62: 559-566, 2015.

25. Jensen KP and Covault J: Human miR-1271 is a miR-96 paralog with distinct non-conserved brain expression pattern. Nucleic Acids Res 39: 701-711, 2011.

26. Yang M, Shan X, Zhou X, Qiu T, Zhu W, Ding Y, Shu Y and Liu P: miR-1271 regulates cisplatin resistance of human gastric cancer cell lines by targeting IGF1R, IRS1, mTOR, and BCL2. Anticancer Agents Med Chem 14: 884-891, 2014.

27. Maurel M, Jalvy S, Ladeiro Y, Combe C, Vachet L, Sagliocco F, Bioulac-Sage P, Pitard V, Jacquemin-Sablon H, Zucman-Rossi J, et al: A functional screening identifies five microRNAs controlling glypican-3: Role of miR-1271 down-regulation in hepatocellular carcinoma. Hepatology 57: 195-204, 2013. 